\title{
Improvement Limitation of Satellite Visibility by Space Diversity Consisted of Two Geostationary Satellites in Urban Areas of Japan
}

\author{
By Toshihiko KITANO Hiroshi JUZOJI Isao NAKAJIMA \\ School of Medicine, Tokai University, Isehara, Japan \\ (Received April 25th, 2008)
}

\begin{abstract}
The paramedic in the moving ambulance sends via the communications satellite the moving picture data to the doctor in the hospital However, when an ambulance runs inside a city, a radio wave to the communication satellite is blocked and as a result the moving picture data sometimes freeze. Maximum value of the satellite visibility by the space diversity which uses two geostationary satellites is acquired in each city of Japan. From these simulation results, it was found that there was a limit in the improvement of the satellite visibility by the space. The limit of this improvement depends on the city. .Moreover the angle of elevation of the quasi zenith satellite which the satellite visibility exceeds the limit value of two geostationary satellites was acquired, and these angles of elevation were compared between each city of Japan. It became clear that more than 69 degrees of an elevation angle of the quasi zenith satellite is necessary to exceed a maximum satellite visibility of two geostationary satellites.
\end{abstract}

Key Words: Satellite Visibility, Space Diversity, Geostationary Satellite, Quasi Zenith Satellite

\section{Introduction}

The objective is to make clear that there is a limit in the improvement of the satellite visibility in the spatial diversity which uses two communications satellites.

The paramedic in the moving ambulance sends via the communications satellite the moving picture data which shows the condition of the patient to the doctor in the hospital However, when an ambulance runs inside a city, a radio wave to the communication satellite is blocked by the building, the roadside tree and the electric light pole which stands along the roadsides, and as a result the moving picture data sometimes freeze.

To improve the probability by which the radio wave is not blocked off, i.e. the satellite visibility, the spatial diversity which uses more than one satellite has been already proposed ${ }^{1)-3}$. Also, it has been already reported that the satellite visibility is more improved by the use of the quasi zenith satellite ${ }^{4)}$. However, the limit of the improvement of the satellite visibility by the spatial diversity consisted of two geostationary satellites has not been so far clarified. The condition of the quasi zenith satellite which exceeds the limit value of the spatial diversity which uses two geostationary satellites has not been so far reported.

In this paper, the hemispherical picture image data which contains a zenith of each Japanese city with the fish-eye camera installed on the roof of the measurement vehicle is acquired. Next, by using this hemispherical picture image data, maximum satellite visibility is acquired fromcombination of all two satellites which are located in any place on the geostationary satellite orbit and a limit value of satellite visibility in each city in Japan is acquired. Moreover, the angle of elevation of the quasi zenith satellite which the satellite visibility exceeds the limit value of the spatial diversity is acquired, and these angles of elevation are compared between each city in Japan. The quasi zenith satellite is composed of more than one orbiting satellite and one of them is always situated near the zenith.

\section{What is Satellite Visibility?}

Fig. 1 is the key map which shows that the radio wave from the satellite is in the blocked condition. As shown in Fig. 1, the blocking obstacle standing along the roadside blocks the radio wave which is propagating via a communication satellite for example a geostationary satellite. In the case of the geostationary satellite the direction of the satellite is constantly fixed in the sky. So the view direction from a running ambulance toward the satellite varies according to change of the ambulance running direction. As a result, the blocking situation to the satellite changes incessantly. The packet communication is a usual system for a satellite communication between an ambulance and a satellite. The packet signal of a moving picture data is lost when the radio wave is blocked. The packet loss results to the loss of picture frame data.

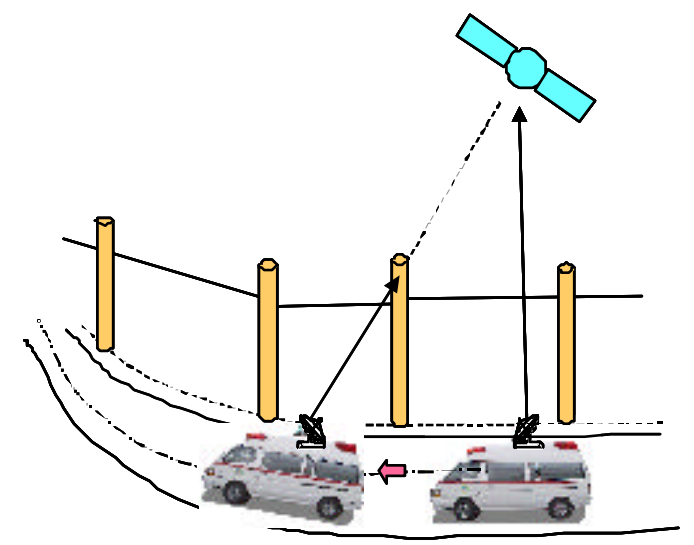

Fig. 1. Key map which shows that the radio wave is blocked. 
The satellite visibility is related to packet loss possibility. The satellite visibility $\mathrm{Vs}$ is defined as one minus the ratio of $\mathrm{Tg}$ and $\mathrm{Tt}$, where the time period $\mathrm{Tt}$ is the total measurement time, and the time period $\mathrm{Tg}$ is the total time during which the satellite is in the blocked state.

Thus, the satellite visibility Vs can be expressed by the following equation.

$$
\mathrm{Vs}=1-\mathrm{Tg} / \mathrm{Tt}
$$

\section{Measurement Method of Satellite Visibility}

The Satellite visibility is measured by use of a fish-eye camera, a GPS sensor and a vehicle speed sensor mounted on the roof of a measuring vehicle ${ }^{5}$. The hemispherical scenery of the urban space is shot as the plane circular picture by the fish-eye. Then, this plane circular picture is recorded in a digital VTR. At the same time, the GPS sensor and the speed sensor, respectively, detect the position and the speed of the running vehicle. Each measuring data is then processed in the separate data processor for making a database. The plane circular picture is processed and converted into a binary frame picture data. Fig. 2 shows the outside appearance of a measuring vehicle.

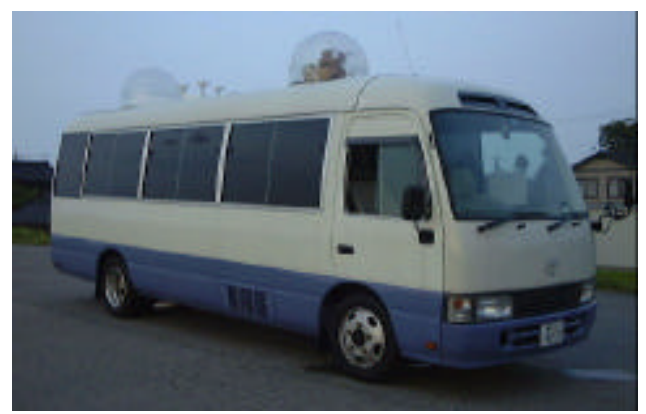

Fig. 2. Outside appearance of measuring vehicle.

The satellite visibility is obtained by using a binary frame picture data transformed from a plane circular picture of the hemispherical urban area. Fig. 3(a) shows a natural plane picture of the hemispherical urban area. Fig. 3(b) shows its binary frame picture data. From binary frame picture data, the blocking object standing along the roadside can be distinguished clearly from sky.

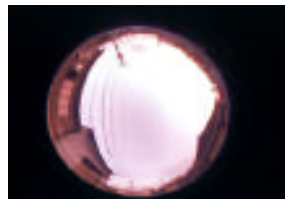

(a) Natural data

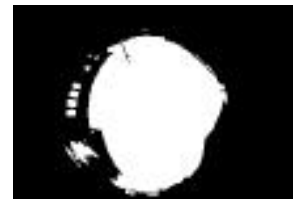

(b) Binary data
Fig. 3. Picture frame data of urban area.

In the binary frame picture data, the zenith corresponds to the center, while the horizon corresponds to the circumference, respectively. The elevation angle corresponds to the radius of a concentric circle. The upward direction corresponds to the running direction of the measuring vehicle. The satellite is displayed as a point. When the measuring vehicle changes its running direction, the point showing the satellite moves in the binary frame picture data according to the running direction of the measuring vehicle.

Fig. 4 shows three explanation figures of the binary frame picture data which show how the satellite is blocked while the measuring vehicle is running. Fig. 4 (a) shows the situation of just before the satellite is blocked. Fig.4 (b) shows the situation of just blocked satellite. Fig.4 (c) shows the situation of just after the satellite was blocked. From Fig.4 (a) to Fig.4 (c), there are multiple binary frame picture data. Therefore, the total numbers of the binary frame picture data are equal to the total of the time period Tt. On the other hand, the total numbers of the binary frame picture data corresponding to the condition that the satellite is blocked are equal to the total of the time period $\mathrm{Tg}$. As a result, the satellite visibility $\mathrm{Vs}$ is expressed by Eq. (1).
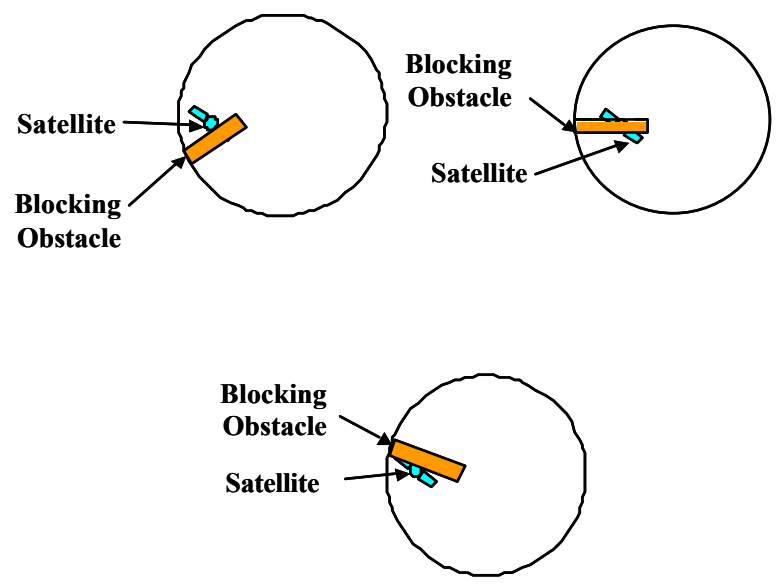

(c)

Fig 4. Binary frame picture illustrations showing how satellite is blocked.

\section{Satellite Visibility by One Geostationary Satellite}

The satellite visibility to the geostationary satellite which can be seen from the measuring vehicle which runs in Japan urban area is obtained by the measurement result of the binary frame picture data in the same area and the calculation result of the geostationary satellite orbit which can see from the same area. The orbit of the geostationary satellite is obtained as follows. The position of the geostationary satellite being at a standstill on a circular orbit above the equator is expressed by the east longitude. The azimuth angle Eaz and the elevation angle Eel to the geostationary satellite which can see from the ground located at east longitude Le and north latitude $\mathrm{La}$, is expressed by the following equations by use 
of east longitude Ls of the geostationary satellite position 6).

$$
\begin{aligned}
& \mathrm{Eel}=\operatorname{Arc} \cos \left(((\mathrm{Ro}+\mathrm{H}) / \mathrm{D}) \cdot \sqrt{1-\cos ^{2}(\mathrm{La}) \cdot \cos ^{2}(\mathrm{Ls}-\mathrm{Le})}\right) \\
& \mathrm{Eaz}=180-\operatorname{Arcsin}\left(\sin (\mathrm{Ls}-\mathrm{Le}) / \sqrt{1-\cos ^{2}(\mathrm{La}) \cdot \cos ^{2}(\mathrm{Ls}-\mathrm{Le})}\right)
\end{aligned}
$$

Wherein, D is expressed by

$$
\mathrm{D}=\longdiv { \mathrm { H } ^ { 2 } - 2 \cdot \mathrm { Ro } \cdot ( \mathrm { H } + \mathrm { Ro } ) \cdot ( 1 - \operatorname { c o s } ( \mathrm { La } ) \cdot \operatorname { c o s } ( \mathrm { Ls } - \mathrm { Le } ) }
$$

, and Ro is the radius of earth, $\mathrm{H}$ is the altitude from the equatorial plane to the geostationary satellite Fig.5 is a calculation result of the orbit of the geostationary satellite which can see from Tokyo Ginza, Sendai City, Hiroshima City.

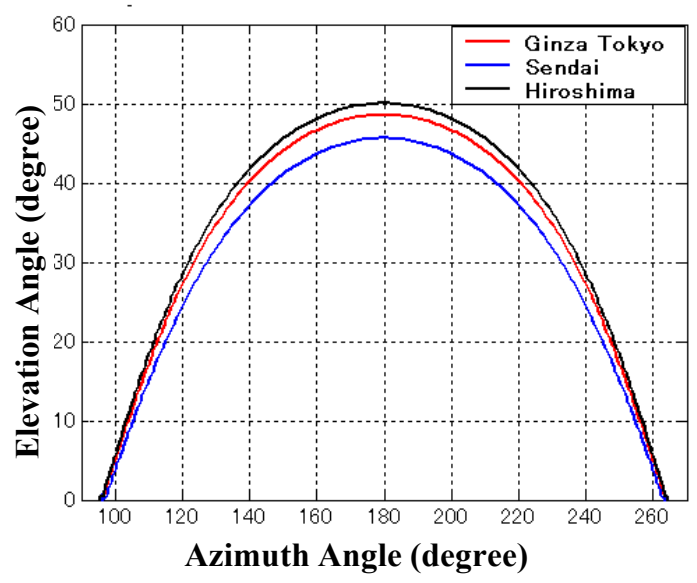

Fig. 5. Orbit of geostationary satellite.

The geostationary satellite orbit which can see from Tokyo Ginza is obtained from the position data( 35.7 degree of north latitude, 138.9 degree of east longitude) and the earth radius Ro $(6378 \mathrm{~km})$ and satellite height $\mathrm{H}$ $(35786 \mathrm{~km})$. The orbit from Sendai City is obtained from the position data (38.3 degree of north latitude, 140.9 degree of east longitude) and $\mathrm{Ro}(6378 \mathrm{~km})$ and $\mathrm{H}(35786$ $\mathrm{km})$. The orbit from Hiroshima City is obtained from the position data (34.4 degree of north latitude, 132.5 degree of east longitude) and Ro $(6378 \mathrm{~km})$ and $\mathrm{H}(35786 \mathrm{~km})$. The transverse of Fig. 5 shows the azimuth angle, i.e. the angle of the horizontal direction to the geostationary satellite. The vertical axis of Fig. 5 shows the elevation angle, i.e. the angle of the vertical direction to the geostationary satellite. In each city, the angle of elevation of the geostationary satellite becomes maximum value at 180 degrees of azimuths. In Fig.5, 180 degrees of azimuths are the southern direction, 90 degrees are the eastern direction and 270 degrees are the western direction.

Fig.6 shows a simulation result of the satellite visibility obtained at Tokyo Ginza, Sendai City, Hiroshima City. Fig.6 is the case which there is one geostationary satellite in the orbit which is shown in Fig.5. Generally, there are only several geostationary satellites in the orbit. However the satellite visibility is acquired based on assumption that one satellite locates in any place on the geostationary satellite. This assumption makes it possible to estimate a position on the orbit of the geostationary satellite that the satellite visibility becomes maximum.

The transverse of Fig. 6 shows the azimuth angle, i.e. the angle of the horizontal direction to the geostationary satellite. The vertical axis of Fig.6 shows the satellite visibility.

The maximum satellite visibility and position of the geostationary satellite, i.e. the azimuth of the geostationary satellite are shown as follows. Tokyo Ginza is maximum satellite visibility $60.9 \%$ at the time of 208 degrees of azimuths. Sendai City is maximum satellite visibility $76.3 \%$ at the time of 171 degrees of azimuths. Hiroshima City is maximum satellite visibility $82.3 \%$ at the time of 185 degrees of azimuths. The azimuth of the geostationary satellite and the maximum satellite visibility are different in each city. This is because the urban area structure for example the height and so on of the blocking obstacle along the roadside which the measurement vehicle runs are different in each city.

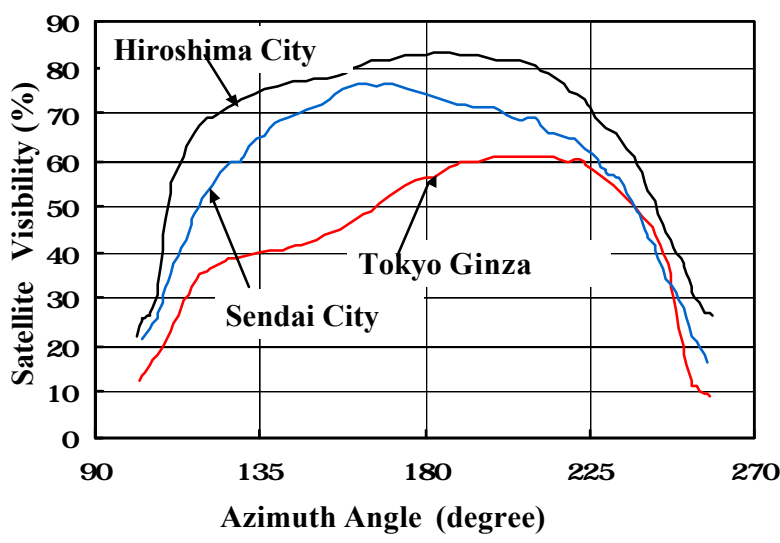

Fig. 6. Satellite visibility of one geostationary satellite case.

\section{Limit of Improvement Effect of Satellite Visibility by Two Geostationary Satellites}

It is well known that the satellite visibility is improved by the spatial diversity of two geostationary satellites. Fig. 7 shows a key map of the space diversity which is realized by the two geostationary satellites. From the measuring vehicle traveling at a position $\mathrm{V} 1$, it is possible to view the geostationary satellite $\mathrm{S} 1$, but it is impossible to view the geostationary satellite $\mathrm{S} 2$ that is being blocked by a utility pole $\mathrm{P} 2$. On the other hand, from the measuring vehicle traveling at a position $\mathrm{V} 2$, it is possible to view the geostationary satellite $\mathrm{S} 2$, but it is impossible to view the geostationary satellite $\mathrm{S} 1$ that is being blocked by a utility pole P2. This fact shows that the satellite visibility to those geostationary satellites can be improved by determining if the geostationary satellites S1 and S2 can be viewed constantly from the traveling measuring vehicle or not. At a time when the geostationary satellite S1 cannot be viewed, the satellite visibility is improved by switching to the geostationary satellite S2.Then at a time when the geostationary satellite $\mathrm{S} 2$ cannot be viewed, 
the satellite visibility is improved by switching to the geostationary satellite $\mathrm{S} 1$.

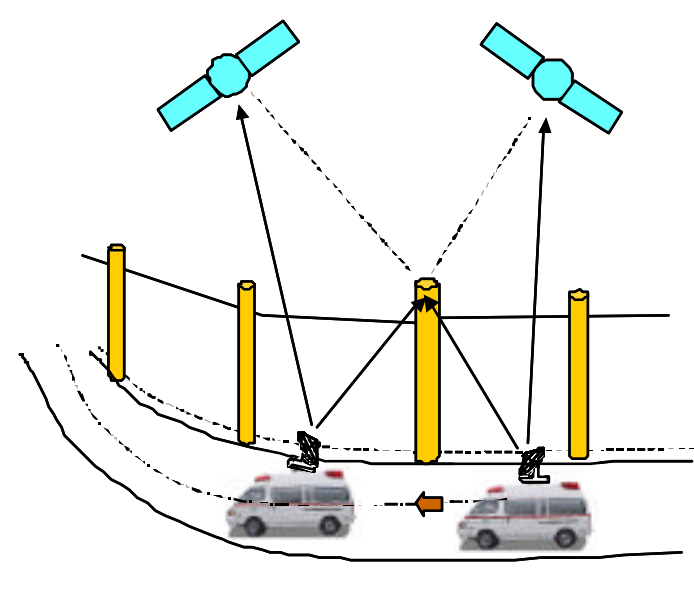

Fig. 7. Key map of space diversity by two geostationary satellites.

The improvement effect of the satellite visibility by space diversity consisted of two geostationary satellites can be confirmed from the measurement result of the binary frame image and the calculation result of the geostationary satellite orbit.

Fig. 8 shows a simulation result of the satellite visibility at Tokyo Ginza when there are two satellites in the geostationary satellite orbit, which is shown in Fig.5.

Two geostationary satellites are situated on the different point on the orbit. When either of two geostationary satellites can be seen from the measurement vehicle that runs along the road, it is defined that the measurement vehicle can see the geostationary satellite. The simulation of Fig.8 was accomplished under this condition. Because two geostationary satellites are situated at the arbitrary point on the orbit, the satellite visibility is obtained at the combination of the position of two geostationary satellites. Therefore, Fig. 8 becomes a three-dimensional chart. The transverse is the azimuth of geostationary satellite S1, the vertical axis is the azimuth of geostationary satellite S2 and the contour line is the satellite visibility. The combination of two geostationary satellites which the satellite visibility becomes maximum can be estimated from Fig. 8. The diagonal line which is shown by the broken line in Fig. 8 is the same as the result which is shown as one geostationary satellite case in Fig.6. When azimuths of the geostationary satellite S1 is 242.1 degrees and azimuths of the geostationary satellite S2 is 189.6 degrees, the maximum satellite visibility becomes $74.8 \%$ at Tokyo Ginza. This value is improved to $13.9 \%$ from $60.9 \%$ of maximums satellite visibility at the time of one geostationary satellite. It becomes the same satellite visibility even if the azimuth of S1 and the azimuth of S2 are exchanged. Therefore, the satellite visibility which is shown in Fig. 8 becomes symmetry against the diagonal line.

Fig. 9 shows a simulation result of the satellite visibility at Sendai City when there are two satellites in the geostationary satellite orbit. When azimuths of the geostationary satellite S1 is 210.0 degrees and azimuths of the geostationary satellite S2 is 139.3 degrees, the maximum satellite visibility becomes $88.6 \%$ at Sendai City. This value is improved to $12.3 \%$ from $76.3 \%$ of maximums satellite visibility at the time of on geostationary satellite.

Fig.10 shows a simulation result of the satellite visibility at Hiroshima City when there are two satellites in the geostationary satellite orbit. When azimuths of the geostationary satellite S1 is 208.2 degrees and azimuths of the geostationary satellite S2 is 110.8 degrees, the maximum satellite visibility becomes $92.8 \%$ at Hiroshima City. This value is improved to $10.5 \%$ from $82.3 \%$ of maximums satellite visibility at the time of one geostationary satellite. As for the improvement of the satellite visibility by the spatial diversity of 2 fixed satellites, it is obvious that there is a limit by above result. This is caused by the urban area structure. It is impossible to improve further satellite visibility, which is due to the use of the geostationary satellite. Above results are shown in Table. 1.

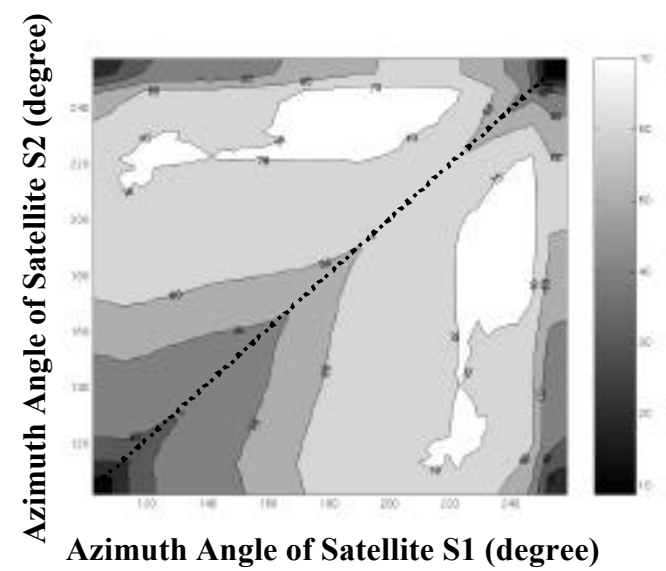

Fig. 8. Simulation result of two geostationary satellites cases at Tokyo Ginza.

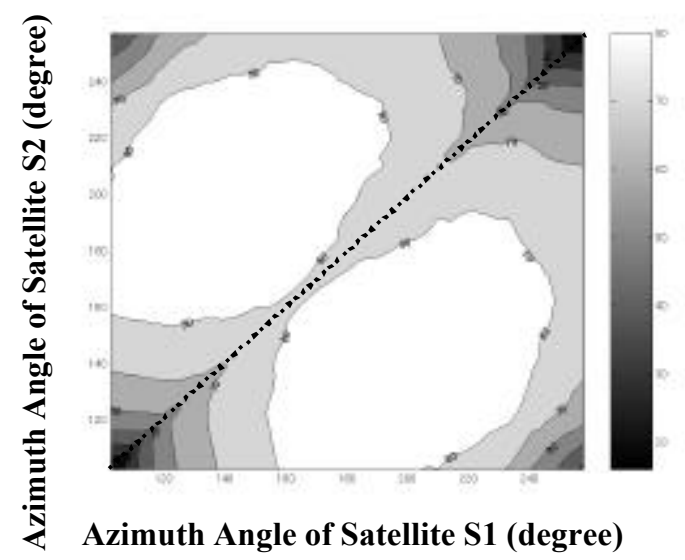

Fig .9. Simulation result of two geostationary satellites cases at Sendai City. 


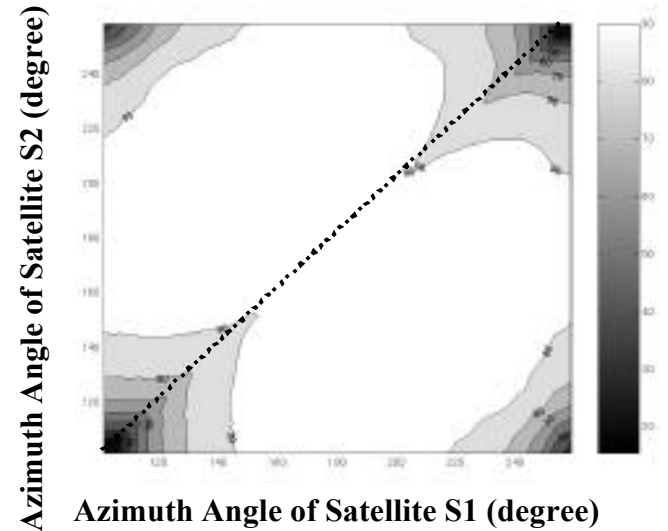

Fig. 10. Simulation result of two Geostationary satellites cases at Hiroshima City.

Table 1. Comparison of satellite visibility between one satellite case and two satellites cases.

\begin{tabular}{|c|c|c|c|c|c|c|}
\hline \multirow[b]{2}{*}{ Cit } & \multicolumn{3}{|c|}{ Two geostationary Satellites } & \multicolumn{2}{|c|}{ One geostationart Satellite } & \multirow{2}{*}{$\begin{array}{l}\text { Improvement } \\
\text { Value of Satellit } \\
\text { Visibility } \\
(\%)\end{array}$} \\
\hline & $\begin{array}{l}\text { Azimuth } \\
\text { Angle of S1 } \\
\text { (degree) }\end{array}$ & \begin{tabular}{|c} 
Azimuth \\
Angle of S2 \\
(degree)
\end{tabular} & $\begin{array}{c}\text { Satellite } \\
\text { Visibility } \\
(\%)\end{array}$ & $\begin{array}{l}\text { Azimuth } \\
\text { Angle } \\
\text { (degree) }\end{array}$ & $\begin{array}{l}\text { Satellitite } \\
\text { Vsibility } \\
(\%)\end{array}$ & \\
\hline Ginza Tokyo & 242.1 & 189.6 & 74.8 & 207.5 & 60.9 & 13.9 \\
\hline Sendai City & 210 & 139.3 & 88.6 & 171 & 76.3 & 12.3 \\
\hline Hiroshima City & 208.2 & 110.8 & 92.8 & 185 & 82.3 & 10.5 \\
\hline
\end{tabular}

From Tab.1 it was found that there was a limit in the improvement of the satellite visibility by the space diversity in two geostationary satellites. The limit of this improvement depends on the city.

\section{Discussion}

The use of the quasi zenith satellite as the way of more improving the satellite visibility has been reported. The quasi zenith satellite is the system to have always staying one orbiting satellite at the upper part of the sky by using more than one orbiting satellite. When the orbiting satellite exists at an arbitrary point in the upper sky the satellite visibility can be obtained by the simulation using the binary frame picture data measured in each city.

Fig.11 is the simulation result of the satellite visibility of Tokyo Ginza against the satellite at an arbitrary azimuth angle and an arbitrary elevation angle. Because the satellite is not a geostationary satellite, the satellite never stops at the arbitrary angle of elevation and the arbitrary azimuth angle. However, one satellite within more than one orbiting satellite is sufficiently thought of about being situated on this position. Fig. 11 is a simulation result of Tokyo Ginza. The transverse shows the angle of elevation value from 60 degrees to 90 degrees and the vertical axis shows the satellite visibility. The azimuth angle is shown as a parameter such as 0 degree, 90 degrees, 180 degrees, 270 degrees. The parallel broken line which is parallel to the transverse of Fig. 11 is satellite visibility maximum $74.8 \%$ of two geostationary satellites case described before. Also, the broken line which is parallel to the vertical axis of Fig.11, indicates the elevation angle of the quasi zenith satellite which exceeds the satellite visibility of maximum value $74.8 \%$ of two geostationary satellites case. If the angle of elevation of the quasi zenith satellite is more than 66.4 degrees at Tokyo Ginza, more than $74.8 \%$ of satellite visibility of two geostationary satellites can be realized in case of the quasi zenith satellite.

Fig.12 is a simulation result of Sendai City. If the angle of elevation of the quasi zenith satellite is more than 69.4degrees at Sendai City, more than $88.6 \%$ of satellite visibility of two geostationary satellites can be realized in case of the quasi zenith satellite.

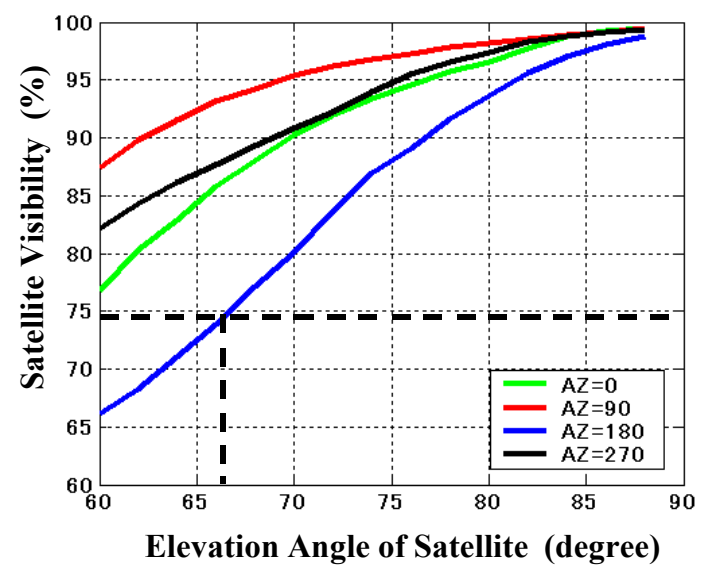

Fig. 11. Simulation result of quasi zenith satellite use at Tokyo Ginza.

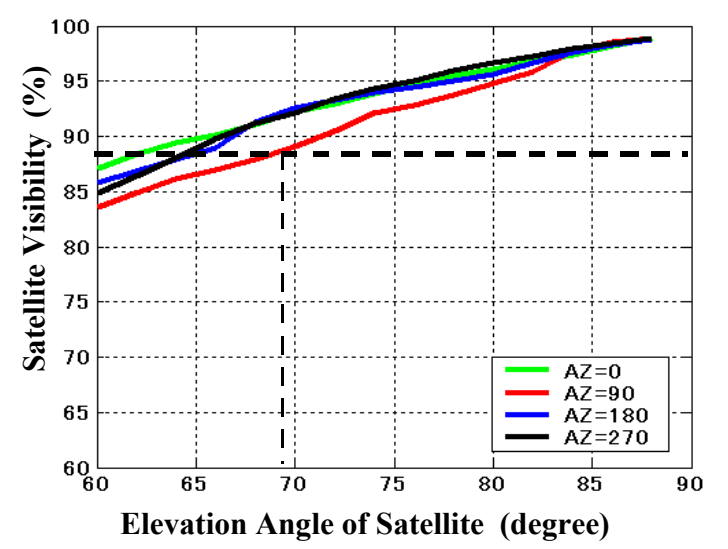

Fig. 12. Simulation result of quasi zenith satellite use at Sendai City. 


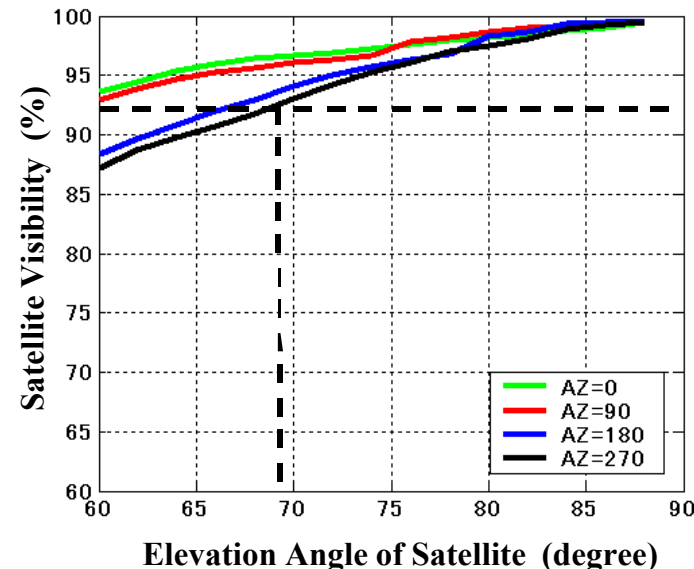

Fig. 13. Simulation result of quasi zenith satellite use at Hiroshima City.

Fig. 13 is a simulation result of Sendai City. If the angle of elevation of the quasi zenith satellite is more than 69.4 degrees at Sendai City, more than $92.8 \%$ of satellite visibility of two geostationary satellites can be realized in case of the quasi zenith satellite.

As a result, it became clear that more than 69 degrees of an elevation angle of the quasi zenith satellite is necessary to exceed a maximum satellite visibility of two geostationary satellites in the urban areas of Japan.

\section{Conclusion}

The paramedic in the moving ambulance sends via the communications satellite the moving picture data which shows the condition of the patient to the doctor in the hospital However, when an ambulance runs inside a city, a radio wave to the communication satellite is blocked by the building, the roadside tree and the electric light pole which stands along the roadsides, and as a result the moving picture data sometimes freeze.

Maximum value of the satellite visibility by the space diversity consisted of two geostationary satellites is acquired by simulation from combination of all two satellites which are located in any place on the geostationary satellite orbit in each city of Japan.

From these simulation results, it was found that there was a limit in the improvement of the satellite visibility by the space diversity which uses two geostationary satellites. The limit of this improvement depends on the city.

Moreover, the angle of elevation of the quasi zenith satellite which the satellite visibility exceeds the limit value of two geostationary satellites was acquired, and these angles of elevation were compared between each city of Japan. As a result, it became clear that more than 69 degrees of an elevation angle of the quasi zenith satellite is necessary to exceed a maximum satellite visibility of two geostationary satellites.

\section{References}

1) Hatsuda, T. Hashimoto and K. Masuda, J.: Diversity Systems Comparison of Satellite Visibility Improvement for Designing Mobile Broadcasting Satellite System, IEEE Trans. on Antenna Propagation, 54 (2006), pp. 2365-2370.

2) Karasawa, Y. Kimura, K. and Minamisono, K.: Analysis of Availability Improvement in LMSS by Means of Satellite Diversity Based on Three-State Propagation Cannel Mode, IEEE Transaction on Vehicle Technology, 46(1997), pp. 10471056.

3) Kitano, T. Tomioka, Y. Juzoji, H. and Nakajima, I.: Improvement of Satellite Visibility by Space Diversity consisted of Two Geostationary Satellites, IEEE $9^{\text {th }}$ International Conference on e-Health Networking Application \&Services, 2007, pp. 137-143.

4) N Obara, N. Ikeda and M. Miura, A.: COMETS on an inclined elliptic orbit -toward quasi-zenith satellite system, Technical Report of IECE, AP2000-59, SAT2000-56, 2000, pp. 7-12.

5) Nakajima, I. Juzoji, H. and Nawaz M. N., PAKISTAN Abstract Books, 2006, pp. 72-78.

6) Kouda, H. and Kondo, k.: Introduction of Practical Design for Geostationary Satellite (in Japanese), JATEC Publication, 1987, pp. 54-56. 